[14] C. M. Ringel, Tame Algebras and Integral Quadratic Forms, Lecture Notes in Math. 1099, Springer, 1984

[15] A. S kow roński, Tame triangular matrix algebras over Nakayama algebras, J. London Math Soc. 34 (1986), 245-264.

INSTITUTE OF MATHEMATICS, NICHOLAS COPERNICUS UNIVERSITY Chopina 12/18, 87-100 Toruń, Poland

\section{Homogeneous cohomology manifolds which are inverse limits}

by

\author{
W. Jakobsche (Warszawa)
}

Abstract. We describe a class of homogeneous cohomology manifolds.

1. Introduction. The aim of this paper is to consider a certain class of compact finite-dimensional, homogeneous spaces which are inverse limits of topological manifolds. We say that a space $X$ is homogeneous if for every $x, y \in X$ there exists a homeomorphism $h: X \rightarrow X$ with $h(x)=y$. The spaces that we consider depend on an orientable $n$-manifold $L^{n}$ (with possibly empty boundary) and on a countable or finite family $\mathscr{M}^{n}$ of closed orientable manifolds of the same dimension $n$. We will denote them by $X\left(L^{n}, \mathscr{M}^{n}\right)$. A first such space was constructed in [J] for $L^{3}=S^{3}$ and the one-element family $\mathscr{M}^{3}=\{H\}$ where $H$ was a homotopy 3 -sphere $\neq S^{3}$, as a potential counterexample to the Bing-Borsuk conjecture $\left({ }^{1}\right)$. Earlier similarly constructed spaces were considered in a different context in [P] and [W]. Then Ancel and Siebenmann ([A-S]) noticed that $X\left(S^{3}, H^{\prime}\right)$ where $H^{\prime}$ is some homology 3-sphere can be identified with a compactification of the Davis contractible 4-manifold which covers a closed 4-manifold [D]. They also introduced axioms describing spaces $X\left(L^{n},\{M\}\right)$ for the families $\mathscr{M}^{n}=\{M\}$ consisting of one element. Axiomatic description seems particularly useful when applied to spaces $X\left(L^{n}, \mathscr{M}^{n}\right)$ with an infinite family $\mathscr{M}$. Our axioms for general spaces $X\left(L^{n}, \mathscr{M}^{n}\right)$ presented in Section 2 differ only slightly from those of Ancel and Siebenmann. They play an important role in the proof of $m$-homogeneity of $X\left(L^{n}, \mathscr{M}^{n}\right)$ given in Sections 7 and 8 . In Section 4 we use a lemma proved by Toruńczyk to show that the spaces $X\left(L^{n}, \mathscr{M}^{n}\right)$ depend only on $L^{n}$ and $\mathscr{M}^{n}$, and in Section 5 we give a construction of $X\left(L^{n}, \mathscr{M}^{n}\right)$. If a family $\mathscr{M}^{n}$ consists of homology $n$-spheres then $X\left(L^{n}, \mathscr{M}^{n}\right)$ is a cohomology manifold. In this case $X\left(L^{n}, \mathscr{M}^{n}\right)$ can often be identified with the fixed-point set of a topological action on a manifold or a cohomology manifold. The theory of such actions was developed in [B]. Such homogeneous cohomology manifolds also appear as compactifications of contractible 4-manifolds, or orbit spaces of actions of 0-dimensional infinite compact groups. We give some examples in Sections 9 and 10

${ }^{1}$ ) I have been informed by several people that J. Martin also considered a similar construction. 
The spaces $X\left(L^{n}, \mathscr{M}^{n}\right)$ have many properties of manifolds besides being homogeneous and being cohomology manifolds if $\mathscr{M}^{n}$ consists of homology spheres. In [J-R] for example, it is shown that they have certain general position properties for $n=3$. Finally, there are many such spaces: in Section 11 it is shown that $X\left(L^{3}, \mathscr{M}\right)$ $\neq X\left(L^{3}, \mathscr{M}^{\prime}\right)$ if $\mathscr{M}$ and $\mathscr{M}^{\prime}$ are infinite families consisting of irreducible 3 -manifolds and $\mathscr{M} \neq \mathscr{M}^{\prime}$. In particular, there exists an uncountable family of non-homeomorphic homogeneous 3-dimensional cohomology manifolds $X\left(S^{3}, \mathscr{M}^{3}\right)$.

2. Axiomatic description of $X\left(L^{n}, \mathscr{M}^{n}\right)$. Let $L^{n}$ be an orientable $n$-manifold with (possibly empty) boundary $\partial L^{n}$ and let $\mathscr{M}^{n}=\left\{M_{1}, M_{2}, \ldots\right\}$ be a finite or countable family of closed, distinct $n$-manifolds. We define a class $X\left(L^{n}, \mathscr{M}^{n}\right)$ of compact spaces as follows: $X \in X\left(L^{n}, \mathscr{M}^{n}\right)$ if and only if $X=\lim _{\leftarrow}\left\{L_{i}, \alpha_{i, i+1}\right\}$ and the spaces $L_{i}$ and maps $\alpha_{i, i+1}: L_{i+1} \rightarrow L_{i}, i \in N$, satisfy the following axioms:

(1) $L_{1}=L^{n}$ and every $L_{i}$ is a connected sum of $L^{n}$ and finitely many $n$-manifolds, each homeomorphic to some member of $\mathscr{M}^{n}$, with $\partial L_{i}=\partial L^{n}$.

(2) There exists a finite collection $\Omega_{i}$ of pairwise disjoint bicollared $n$-cells in $L_{i} \backslash \partial L_{i}$.

(3) $\alpha_{i, i+1}: L_{i+1} \rightarrow L_{i}$ is a homeomorphism over the set $L_{i} \backslash \bigcup\left\{\right.$ int $\left.Y: Y \in \Omega_{i}\right\}$.

(4) For every $Y \in \Omega_{i}, \alpha_{i, i+1}^{-1}(Y)$ is homeomorphic to $M \backslash D^{n}$, where $M \in \mathscr{M}^{n}$ and $D^{n}$ is a bicollared disk in $M$.

(5) For every $j>i$ if $Y \in \Omega_{i}$ and $Y^{\prime} \in \Omega_{j}$, then $\partial Y \cap \alpha_{i j}\left(Y^{\prime}\right)=\varnothing$ (here $\alpha_{i j}$ $=\alpha_{i, i+1} \circ \ldots \circ \alpha_{j-1, j}: L_{j} \rightarrow L_{i}$. We also put $\alpha_{i, i}=\mathrm{id}_{L_{i}}$ ).

(6) The collection of sets $\left\{\alpha_{i j}(Y): j \geqslant i, Y \in \Omega_{j}\right\}$ is a null family, that is, for every $\varepsilon>0$, only a finite number of elements of the family have diameter $\geqslant \varepsilon$.

(7) The sum $\bigcup \Omega_{i}$ of the collection of cells $\Omega_{i}=\left\{\alpha_{i j}(Y): j \geqslant i, Y \in \Omega_{j}\right.$ and $\alpha_{i j}(Y)$ is not contained in $\alpha_{i k}\left(Y^{\prime}\right)$ for any $i \leqslant k<j$ and $\left.Y^{\prime} \in \Omega_{k}\right\}$ is dense in $L_{i}$.

(8) For $M \in \mathscr{M}^{n}$ let $\Omega^{\prime}(M)=\left\{\alpha_{i j}(Y) \in \Omega_{i}: Y \in \Omega_{i}\right.$ and $\left.\alpha_{j, j+1}^{-1}(Y) \approx M \backslash D^{n}\right\}$. Then the sum $\bigcup \Omega^{\prime}{ }_{i}\left(M_{j}\right)$ of $\Omega^{\prime}{ }_{i}\left(M_{j}\right)$ is dense in $L_{i} \backslash \bigcup_{k \in N \backslash\{j\}}\left(\bigcup \Omega^{\prime}{ }_{i}\left(M_{k}\right)\right)$, for every $i, j \in N$.

We will denote by $\alpha_{i}: X \rightarrow L_{i}$ the natural inverse limit projection.

The axiomatic description of $X\left(L^{n}, \mathscr{M}^{n}\right)$ presented here was first given by Ancel and Siebenmann for a one-element family $\mathscr{M}^{n}=\{M\}$. The families $\Omega^{\prime}{ }_{i}$ and $\Omega^{\prime}{ }_{j}\left(M_{i}\right)$ defined in axioms (7) and (8) are determined by $\Omega_{i}$, so the spaces in $X\left(L^{n}, \mathscr{M}^{n}\right)$ depend on $\mathscr{M}^{n}$, the spaces $L_{i}$, the maps $\alpha_{i, i+1}$ and the families $\Omega_{i}$. By a defining system for $X$ we will mean a family $\left\{\mathscr{M}^{n}, L_{i}, \alpha_{i, i+1}, \Omega_{i}\right\}$ such that $X=\lim _{\leftarrow}\left\{L_{i}, \alpha_{i, i+1}\right\}$. In Section 4 we will show that there is only one space $X \in X\left(L^{n}, \mathscr{M}^{n}\right)$, so we will often write $X=X\left(L^{n}, \mathscr{M}^{n}\right)$. This will show that $X$ depends only on $L_{n}$ and $\mathscr{M}^{n}$, no matter which defining system for $X$ we choose.

Let us note the following:

Proposition (2.1). If every $M \in \mathscr{M}^{n}$ is a homology n-sphere, then $X \in X\left(L^{n}, \mathscr{M}^{n}\right)$ is a cohomology n-manifold.

Proof. In Section 8 we will prove that $X \in X\left(L^{n}, \mathscr{M}^{n}\right)$ is homogeneous so it is enough to compute the local Betti numbers $p^{i}(x, X)$ (see [B], pp. 7-9) around $x=\bigcap_{k=1}^{\infty} \alpha_{i_{k}}^{-1}\left(Y_{i_{k}}\right)$ where $\alpha_{i}: X \rightarrow L_{i}$ is the inverse limit projection, $Y_{i_{k}}$ is an element of some $\Omega_{i_{k}}$ and $\left\{i_{k}\right\}$ is a sequence such that $\alpha_{i_{k}, i_{k+1}}\left(Y_{i_{k+1}}\right) \subset Y_{i_{k}}$. By continuity

$$
\breve{H}^{i}\left(X, X \backslash \alpha_{i_{k}}^{-1}\left(\stackrel{\circ}{Y}_{i_{k}}\right)\right) \approx \varliminf H^{i}\left(L_{j}, L_{j} \backslash \alpha_{i_{k}, j}^{-1}\left(\stackrel{\Upsilon}{i}_{i_{k}}\right)\right)
$$

and these groups are 0 for $i \neq n, 0$ and $Z$ for $i=0$ or $n$. It is easy to see that the natural homomorphism $j_{k l}^{i}: \breve{H}^{i}\left(X, X \backslash \alpha_{i_{k}}^{-1}\left(\dot{Y}_{i k}\right)\right) \rightarrow \breve{H}^{i}\left(X, X \backslash \alpha_{i_{l}}^{-1}\left(\dot{Y}_{i_{l}}\right)\right)$ is an isomorphism, so $p^{i}(x, X)=0$ for $i \neq n, 0$ and $p^{n}(x, X)=1 . X$ is locally orientable, so by [B], p. 9 , it is a cohomology manifold.

Proposition (2.2). If $X \in X\left(L^{n}, \mathscr{M}^{n}\right)$, then $\operatorname{dim} X=n$.

Proof is the same as in [J], p. 134.

3. Auxiliary lemma. Let $\mathscr{Z}$ be a family of $n$-cells contained in the interior of a given $n$-manifold $M$. By $S(\mathscr{L})$ we will denote the sum of interiors of all $n$-cells $Z \in \mathscr{Z}$. We assume that we have fixed an orientation on $M$ and the induced orientation on $\partial M$, and also on $Z$ and $\partial Z$ for every $Z \in \mathscr{Z}$.

We will say that a countable family $\mathscr{Z}$ of $n$-cells in the interior of a given $n$-manifold $M$ is a good stratified family if the following conditions are satisfied:

(1) $\mathscr{Z}=\mathscr{Z}_{1} \cup \mathscr{Z}_{2} \cup \ldots$, where each $\mathscr{Z}_{i}$ is a countable subfamily of $\mathscr{Z}$, and there is a countable or finite number of the families $\mathscr{Z}_{i}$.

(2) Each $S\left(\mathscr{Z}_{i}\right)$ is dense in $M \backslash S\left(\mathscr{Z}_{1} \cup \ldots \cup \mathscr{Z}_{i-1} \cup \mathscr{Z}_{i+1} \cup \ldots\right)$.

(3) $\partial Z$ is bicollared in $M$ for every $Z \in \mathscr{Z}$.

(4) For every $Z_{1}, Z_{2} \in \mathscr{Z}, Z_{1} \neq Z_{2}$, we have $Z_{1} \cap Z_{2}=\varnothing$.

(5) $\mathscr{Z}$ is a null family in $M$, i.e. for every $\varepsilon>0$ the set $\{Z \in \mathscr{Z}: \operatorname{diam} Z>\varepsilon\}$ is finite.

The following lemma (and its proof) is a simple extension of Toruńczyk's Lemma from $[\mathrm{J}]$

LeMMA (3.1). Let $M$ and $N$ be orientable n-manifolds and let $h: M \rightarrow N$ be an orientation-preserving homeomorphism. Let $\mathscr{Y}=\mathscr{Y}^{1} \cup \mathscr{Y}^{2} \cup \ldots$ and $\mathscr{Z}=\mathscr{Z}^{1} \cup \mathscr{Z}^{2} \cup \ldots$ be two good stratified families (each containing the same number, finite or infinite, of subfamilies) of $n$-cells in the interior of $M$ and $N$ respectively. For every $(Y, Z) \in \mathscr{Y}^{i} \times \mathscr{Z}^{i}$ let $\varphi_{Y}^{Z}: \partial Y \rightarrow \partial Z$ be an orientation-preserving homeomorphism. Then there exist bijective functions $p_{i}: \mathscr{Y}^{i} \rightarrow \mathscr{Z}^{i}$ and a homeomorphism $h^{\prime}: M \backslash S(\mathscr{Y}) \rightarrow N \backslash S(\mathscr{Z})$ such that $h^{\prime}|\partial M=h| \partial M$ and $h^{\prime} \mid \partial Y=\varphi_{Y}^{P^{(Y)}}$ for every $Y \in \mathscr{O}^{i}$ and $i \in N$.

We set $p=\bigcup_{i=1}^{\infty} p_{i}: \mathscr{Y} \rightarrow \mathscr{Z}$.

Sketch of the proof. We assume (without loss of generality) that $M=N$, $h=\operatorname{id}_{M}$ and $\operatorname{diam} M \leqslant 1$. Each $\varphi_{Y}^{Z}$ can be extended to a homeomorphism $\psi_{Y}^{Z}: Y \rightarrow Z$ for $Y \in \mathscr{Y}^{i}$ and $Z \in \mathscr{Z}^{i}, i \in N$. Let $\Psi_{i}=\left\{\psi_{Y}^{Z}: Y \in \mathscr{Y}^{i}, Z \in \mathscr{Z}^{i}\right\}$, and let $H(M)$ be the set of all homeomorphisms of $M$ which are identity on $\partial M$. Set $\mathscr{Z}_{n}^{i}=\left\{Z \in \mathscr{Z}^{i}: \operatorname{diam} Z \geqslant 2^{-n}\right\}$, $\mathscr{Y}_{n}^{i}=\left\{Y \in \mathscr{Y}^{i}: \operatorname{diam} Y \geqslant 2^{-n}\right\}$,

$$
\mathscr{Z}_{n}=\bigcup_{i=1}^{\infty} \mathscr{Z}_{n}^{i}, \quad \mathscr{Y}_{n}=\bigcup_{i=1}^{\infty} \mathscr{Y}_{n}^{i}
$$

For any $f \in H(M)$ and any family $\mathscr{T}$ of subsets of $M$, let $f(\mathscr{T})=\{f(T): T \in \mathscr{T}\}$. By (5), $\mathscr{Z}_{n}$ and $\mathscr{Y}_{n}$ are finite families. We construct inductively homeomorphisms $f_{n}, g_{n} \in H(M)$, 
$n=1,2, \ldots$, such that the following conditions are satisfied:

$\left(a_{n}\right)$ If $Y \in \mathscr{Y}_{n}^{i}$ then there is $Z \in \mathscr{Z}^{i}$ such that $f_{n}(Y)=g_{n}(Z)$ and $g_{n}^{-1} f_{n} \mid Y \in \Psi_{i}$ for every $i$

$\left(a_{n}\right)^{\prime}$ If $Z \in \mathscr{Z}_{n}^{i}$ then there is $Y \in \mathscr{Y}^{i}$ such that $f_{n}(Y)=g_{n}(Z)$ and $g_{n}^{-1} f_{n} \mid Y \in \Psi_{i}$ for every $i$.

(b $\left.b_{n}\right) \operatorname{diam} f_{n}(Y)<2^{-n}$ for every $Y \in \mathscr{Y} \backslash\left(\mathscr{Y}_{n} \cup f_{n}^{-1} g_{n}\left(\mathscr{Z}_{n}\right)\right)$.

$\left(\mathrm{b}_{n}\right)^{\prime} \operatorname{diam} g_{n}(Z)<2^{-n}$ for every $Z \in \mathscr{Z} \backslash\left(\mathscr{Z}_{n} \cup g_{n}^{-1} f_{n}\left(\mathscr{Y}_{n}\right)\right)$

(c $\left.c_{n}\right) f_{n}\left|Y=f_{n-1}\right| Y$ for every $Y \in \mathscr{Y}_{n-1} \cup f_{n-1}^{-1} g_{n-1}\left(\mathscr{Z}_{n-1}\right)$.

$\left(\mathrm{c}_{n}\right)^{\prime} g_{n}\left|Z=g_{n-1}\right| Z$ for every $Z \in \mathscr{Z}_{n-1} \cup g_{n-1}^{-1} f_{n-1}\left(\mathscr{Y}_{n-1}\right)$.

$\left(\mathrm{d}_{n}\right) \operatorname{dist}\left(f_{n}, f_{n-1}\right) \leqslant 2^{-n+2}, \operatorname{dist}\left(f_{n}^{-1}, f_{n-1}^{-1}\right) \leqslant 2^{-n+2}$.

$\left(\mathrm{d}_{n}\right)^{\prime} \operatorname{dist}\left(g_{n}, g_{n-1}\right) \leqslant 2^{-n+3}, \operatorname{dist}\left(g_{n}^{-1}, g_{n-1}^{-1}\right) \leqslant 2^{-n+3}$.

The construction is exactly the same as in [J], pp. 129-130, so we omit it. The only difference is that we have to choose the elements $Z_{Y}$ and $Y_{Z}[\mathrm{~J}]$ (p. 130) in the appropriate families $\mathscr{Z}^{i}$ or $\mathscr{Y}^{i}$. We can also use the annulus theorem for any dimension by [K] and [Q]. Having $f_{n}$ and $g_{n}$ we put $f=\lim f_{n}, g=\lim g_{n}$; they are both in $H(M)$, and $h^{\prime \prime}=g^{-1} f$ is a homeomorphism such that $h^{\prime \prime}(M \backslash S(\mathscr{Y}))=M \backslash S(\mathscr{Z}), h^{\prime \prime}\left(S\left(\mathscr{Y}^{\prime}\right)\right)=S\left(\mathscr{Z}^{i}\right)$ for $i=1,2, \ldots$, and $h^{\prime \prime} \mid Y \in \Psi_{i}$ for every $Y \in \mathscr{Y}^{i}$. So we can take $h^{\prime}=h^{\prime \prime} \mid M \backslash S(\mathscr{Y})$ and $p(Y)=h^{\prime \prime}(Y)$ for every $Y \in \mathscr{Y}$.

4. The uniqueness of $X \in X\left(L^{n}, \mathscr{M}^{n}\right)$. Let $X \in X\left(L^{n}, \mathscr{M}^{n}\right)$ where $\mathscr{M}^{n}=\left\{M_{1}, M_{2}, \ldots\right\}$ and let $\left\{\mathscr{M}^{n}, L_{i}, \alpha_{i, i+1}, \Omega_{i}\right\}$ be a defining system for $X$. We set $\mathscr{Y}^{\varnothing}=\mathscr{Y}_{1}^{\varnothing} \cup \mathscr{Y}_{2}^{\varnothing} \cup \ldots=\Omega^{\prime}{ }_{1}$ where $Y_{j}^{\varnothing}=\Omega^{\prime}{ }_{1}\left(M_{j}\right)$. Let us index the elements of the family $\mathscr{Y}^{\varnothing}: \mathscr{Y}^{\varnothing}=\left\{Y_{i_{1}}\right\}_{i_{1} \in N}$, and for every index $i_{1} \in N$, let us define integers $j_{1}=j_{1}\left(i_{1}\right)$ and $k_{1}=k_{1}\left(i_{1}\right)$ as follows: $j_{1}$ is an integer such that $Y_{i_{1}} \in \mathscr{Y}_{j_{1}}^{\varnothing}$ and $k_{1}$ is the uniquely determined integer such that $\alpha_{1, k_{1}}^{-1}\left(Y_{i_{1}}\right) \in \Omega_{k_{1}}$, or equivalently, $\alpha_{1, k_{1}}^{-1}\left(Y_{i_{1}}\right) \approx D^{n}$ and $\alpha_{1, k_{1}+1}^{-1}\left(Y_{i_{1}}\right) \approx M_{j_{1}} \backslash D^{\circ}$.

Now we make the following:

Inductive assumption. Let $\mathscr{I}=\left\{i_{1}, \ldots, i_{m}\right\}$ be a finite sequence of positive integers and that with every $l \leqslant m$ we have associated two positive integers

$$
j_{l}=j_{l}\left(i_{1}, \ldots, i_{l}\right) \quad \text { and } \quad k_{l}=k_{l}\left(i_{1}, \ldots . i_{l}\right)
$$

so that $1 \leqslant k_{1}<\ldots<k_{m}$, and that there is a sequence of $n$-cells

(4.1) $\quad Y_{i_{1}} \subset L_{1}, \quad Y_{i_{1}, i_{2}} \subset \alpha_{1, k_{1}+1}^{-1}\left(Y_{i_{1}}\right), \ldots, \quad Y_{i_{1}, \ldots, i_{m}} \subset \alpha_{k_{m}-2, k_{m-1}+1}^{-1}\left(Y_{i_{1}, \ldots, i_{m-1}}\right)$

and that

$$
\begin{aligned}
& \alpha_{1, k_{1}}^{-1}\left(Y_{i_{1}}\right) \in \Omega_{k_{1}}, \quad \alpha_{k_{1}+1, k_{2}}^{-1}\left(Y_{i_{1}, i_{2}}\right) \in \Omega_{k_{2}}, \ldots, \quad \alpha_{k_{m-1}+1, k_{m}}^{-1}\left(Y_{i_{1}, \ldots, l_{m}}\right) \in \Omega_{k_{m}} ; \\
& Y_{i_{1}, \ldots, l_{l}} \in \Omega_{k_{l}}\left(M_{j_{l}}\right) \quad \text { for } l \leqslant m \text {. }
\end{aligned}
$$

With this inductive assumption, we now define a family $\mathscr{Y}^{\mathscr{F}}=\mathscr{Y}_{1}^{\mathscr{F}} \cup \mathscr{Y}_{2}^{\mathscr{f}} \cup \ldots$ by

$$
\mathscr{Y}^{\mathscr{S}}=\left\{Y \in \Omega_{k_{m}+1}^{\prime}: Y \subset \alpha_{k_{m-1}+1, k_{m}+1}^{-1}\left(Y_{i_{1}, \ldots, i_{m}}\right)\right.
$$

and then $\mathscr{Y}_{j}^{\mathscr{f}}=\mathscr{Y}^{\mathscr{S}} \cap \Omega_{k_{m}+1}^{\prime}\left(M_{j}\right)$. We can write $\mathscr{Y}^{\mathscr{S}}=\left\{Y_{i_{1}, i_{2} \ldots, i_{m+1}}\right\}_{i_{m+1} \in N}$, where $i_{1}, \ldots, i_{m}$ are fixed by the inductive assumption, and $i_{m+1} \in N$ is a varying index which distinguish̆es an element of $\mathscr{Y}^{\mathscr{S}}$. We will also use the notation $Y_{\mathcal{S}, i_{m+1}}$ for $Y_{i_{1}, \ldots, i_{m+1}}$ and
$Y_{\mathscr{I}}$ for $Y_{i_{1}, \ldots, i_{m}}$. Then we define numbers

$$
j_{m+1}=j_{m+1}\left(i_{1}, \ldots, i_{m+1}\right) \quad \text { and } \quad k_{m+1}=k_{m+1}\left(i_{1}, \ldots, i_{m+1}\right)
$$

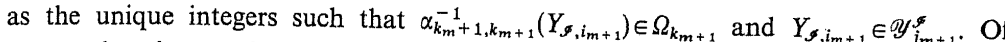
course then $k_{m+1}>k_{m}$, and conditions (4.1-3) are satisfied with $m+1$ in place of $m$.

Starting the induction with $\mathscr{Y}^{\varnothing}$ we get the families $\mathscr{Y}^{\mathscr{F}}=\left\{Y_{\mathscr{F}, i_{m+1}}\right\}_{i_{m+1} \in N}$ of $n$-cells for any finite ordered set $\mathscr{I}=\left\{i_{1}, \ldots, i_{m}\right\}$ of positive integers so that conditions $(4.1-3)$ are satisfied, and for every $Y_{i_{1}, \ldots, i_{m}}$ we have a good stratified family $\mathscr{Y}^{\mathscr{s}}=\mathscr{Y}_{1}^{s} \cup \mathscr{Y}_{2}^{s} \cup \ldots$ of $n$-cells in $\alpha_{k_{m-1}+1, k_{m+1}}^{-1}\left(Y_{i_{1}, \ldots, i_{m}}\right) \approx M_{j_{m}} \backslash D^{D^{n}}$.

We can also define the families

$$
\mathscr{Y}^{m}=\bigcup_{i_{1}, \ldots, i_{m} \in N} \mathscr{Y}^{\left\{i_{1}, \ldots, i_{m}\right\}} \text { and } \mathscr{Y}_{j}^{m}=\bigcup_{i_{1}, \ldots, i_{n} \in N} \mathscr{Y}^{\left\{i_{1}, \ldots, i_{m}\right\}}
$$

of course then $\mathscr{Y}^{m}=\mathscr{Y}_{1}^{m} \cup \mathscr{Y}_{2}^{m} \cup$.

Notice that the members of $\mathscr{Y}^{m}$ are cells which are contained in many different manifolds $L_{i}$.

Then let us consider the family $\mathscr{O S}^{*}=\left\{\alpha_{k_{m-1}+1}^{-1}\left(Y_{i_{1}, \ldots, i_{m}}\right): m \in N, i_{1}, \ldots i_{m} \in N\right\}=\left\{Y_{i_{1}, \ldots, i_{m}}^{*}\right\}$ consisting of closed subsets of $X=\lim _{\leftarrow}\left\{L_{i}, \alpha_{i, i+1}\right\}$. We have

(4.4) $Y_{i_{1}}^{*} \supset Y_{i_{1}, i_{2}}^{*} \supset Y_{i_{1}, i_{2}, i_{3}}^{*} \supset \ldots$

for every infinite sequence of integers $\left\{i_{1}, i_{2}, \ldots\right\}$.

Moreover, by axiom (6), for every such sequence

$$
\tilde{Y}_{\left\{i_{1}, i_{2}, \ldots\right\}}=\bigcap_{l=1}^{\infty} Y_{i_{1}, \ldots, i_{l}}^{*}
$$

is a point.

The set $\tilde{Y}$ consisting of all such intersection points is dense in $X$, with one point corresponding to one sequence $\left\{i_{1}, i_{2}, \ldots\right\}$. Using axiom (3) and the projections $\alpha_{i}: X \rightarrow L_{i}$ to make the necessary identifications in $L_{i}$ 's and $X$ we have the equality

(4.5) $\quad X \backslash \tilde{Y}=\left(L_{1} \backslash S\left(\mathscr{Y}^{\varnothing}\right)\right) \cup \bigcup_{i_{1} \in N}\left(\alpha_{1, k_{1}+1}^{-1}\left(Y_{i_{1}}\right) \backslash S\left(\mathscr{Y}^{\left(i_{1}\right)}\right)\right)$

$$
\cup \bigcup_{i_{1}, i_{2} \in N}\left(\alpha_{k_{1}+1, k_{2}+1}^{-1}\left(Y_{i_{1}, i_{2}}\right) \backslash S\left(\mathscr{Y}^{\left(i_{1}, i_{2} \jmath\right.}\right)\right) \cup \ldots
$$

Here in each summand $k_{m}=k_{m}\left(i_{1}, \ldots, i_{m}\right)$, where $i_{1}, \ldots, i_{m}$ are the integers appearing in this particular summand. Also using the fact that $\alpha_{i} \mid \alpha_{i}^{-1}\left(\partial L_{1}\right): \alpha_{i}^{-1}\left(\partial L_{1}\right) \rightarrow \partial L_{1}$ is a homeomorphism, we can assume that $\partial L=\partial L_{1} \subset X$. Now we prove the following:

Theorem (4.6). Let $X \in X\left(L^{n}, \mathscr{M}^{n}\right)$ and $\bar{X} \in X\left(\bar{L}^{n}, \mathscr{M}^{n}\right)$ and let $h: L^{n} \rightarrow \bar{L}^{n}$ be an orientation-preserving homeomorphism. Then there exists a homeomorphism $h^{*}: X \rightarrow \vec{X}$ such that $h^{*}\left|\partial L^{n}=h\right| \partial L^{n}$.

Proof. Let $\left\{\mathscr{M}^{n}, L_{i}, \alpha_{i, i+1}, \Omega_{i}\right\}$ and $\left\{\mathscr{M}^{n}, \bar{L}_{i}, \bar{\alpha}_{i, i+1}, \bar{\Omega}_{i}\right\}$ be defining sequences for $X$ and $\bar{X}$. For $X$ we will use all the notation established until now, that is we have families $\Omega_{i}^{\prime}, \Omega_{i}^{\prime}\left(M_{j}\right), \mathscr{Y}^{\mathscr{\prime}}, \mathscr{Y}_{j}^{*}, \mathscr{Y}^{m}, \mathscr{Y}_{j}^{m}, \mathscr{Y}^{*}$ and the spaces $Y_{i_{1}, \ldots, i_{m}}, Y_{i, \ldots, i_{m}}^{*}, \tilde{Y}$. Accordingly 
for $\bar{X}$ we have families $\bar{\Omega}_{i}^{\prime}, \bar{\Omega}_{i}^{\prime}\left(M_{j}\right), \mathscr{Z}^{\mathscr{J}}, \mathscr{Z}_{j}^{\mathscr{\Phi}}, \mathscr{Z}^{m}, \mathscr{Z}_{j}^{m}, \mathscr{Z}^{*}$ and spaces $Z_{i_{1}, \ldots, i_{m}}, Z_{i_{1}, \ldots, i_{m}}^{*}$, $\tilde{Z}$ etc.

We want to prove that $X$ and $\bar{X}$ are homeomorphic. First we use Lemma (3.1) with $M=L^{n}=L_{1}, N=\bar{L}^{n}=\bar{L}_{1}$, and the stratified families $\mathscr{y}=\mathscr{y}^{\varnothing}=\mathscr{y}_{1}^{\varnothing} \cup \mathscr{Y}_{2}^{\emptyset} \cup \ldots$ and $\mathscr{Z}=\mathscr{Z}^{\varnothing}=\mathscr{Z}_{1}^{\emptyset} \cup \mathscr{Z}_{2}^{\varnothing} \cup \ldots$ of $n$-cells in $L^{n}$ and $\bar{L}^{n}$ respectively, to find a homeomorphism

$$
h_{1}^{*}: L_{1} \backslash S\left(\mathscr{Y}^{\varnothing}\right) \rightarrow \bar{L}_{1} \backslash S\left(\mathscr{Z}^{\varnothing}\right)
$$

with $h_{1}^{*}\left|\partial L_{1}=h\right| \partial L^{n}$ and bijective functions $p_{j}: \mathscr{Y}_{j}^{\emptyset} \rightarrow \mathscr{Z}_{j}^{\varnothing}$ such that for every $Y \in \mathscr{Y}_{j}^{\varnothing}$, $h_{1}^{*}(\partial Y)=\partial\left(p_{j}(Y)\right)$.

Assume inductively that we have defined a homeomorphism $h_{m}^{*}$ which takes the union of the first $m$ summands of (4.5):

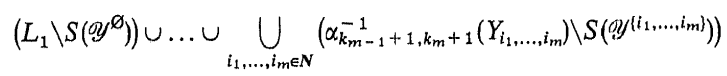

onto the corresponding union

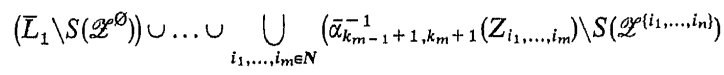

and bijective functions $p_{j}^{m}: \mathscr{Y}_{j}^{m} \rightarrow \mathscr{Z}_{j}^{m}$ so that $h_{m}^{*}\left|\partial L^{n}=h\right| \partial L^{n}$ and for every $Y \in \mathscr{Y}_{j}^{m}$ we have $h_{m}^{*}(\partial Y)=\partial\left(p_{j}^{m}(Y)\right)$; we also denote by $p^{m}$ the map defined by $p^{m}(Y)=p_{j}^{m}(Y)$ for any $Y \in \mathscr{Y}_{j}^{m}$ and any $j \in N$. Take any $Y=Y_{i_{1}, \ldots, i_{m+1}} \in \mathscr{Y}^{m}$, and let $Z=Z_{i_{1}, \ldots, i_{m+1}^{\prime}}=p^{m}(Y)$. Of course $Z \in \mathscr{Z}^{m}$. We have the numbers $j_{m+1}=j_{m+1}\left(i_{j}, \ldots, i_{m+1}\right)$ and $k_{m+1}=k_{m+1}\left(i_{1}, \ldots, i_{m+1}\right)$ defined uniquely for $Y_{i_{1}, \ldots, i_{m+1}}$ and the numbers $j_{m+1}^{\prime}=j_{m+1}^{\prime}\left(i_{1}^{\prime}, \ldots, i_{m+1}^{\prime}\right)$ and $k_{m+1}^{\prime}=k_{m+1}^{\prime}\left(i_{1}^{\prime}, \ldots, i_{m+1}^{\prime}\right)$ defined for $Z_{i_{1}, \ldots, i_{m+1}}$. By the definition of $p^{m}$ as $p^{m}=\bigcup p_{j}^{m}$, we have $j_{m+1}=j_{m+1}^{\prime}$. Let $\mathscr{I}=\left\{i_{1}, \ldots, i_{m+1}\right\}$ and $\mathscr{I}^{\prime}=\left\{i_{1}^{\prime}, \ldots, i_{m+1}^{\prime}\right\}$.

Now we use Lemma (3.1) again with $M=\alpha_{k_{m}+1, k_{m+1}+1}^{-1}(Y)$ and $N=\bar{\alpha}_{k_{m}^{\prime}+1, k_{m+1}^{\prime}+1}^{-1}(Z)$. Because $j_{m+1}=j_{m+1}^{\prime}$, we have a homeomorphism $h: M \rightarrow N$, and we have good stratified families $\mathscr{Y}^{\mathscr{F}}=\mathscr{Y}_{1}^{\mathscr{F}} \cup \mathscr{Y}_{2}^{\mathscr{F}} \cup \ldots$ and $\mathscr{Z}^{\mathscr{\mathscr { F }}}=\mathscr{Z}_{1} \cup \mathscr{Z}_{2} \cup \ldots$ We can also assume that $h\left|\partial M=h_{m}^{*}\right| \alpha_{k_{m}+1, k_{m+1}+1}^{-1}(\partial Y)$. Now using Lemma (3.1) we get a homeomorphism

$$
h_{\mathscr{F}}^{*}: \alpha_{k_{m}+1, k_{m+1}+1}^{-1}(Y) \backslash S\left(\mathscr{Y}^{\mathscr{J}}\right) \rightarrow \bar{\alpha}_{k_{m}^{\prime}+1, k_{m+1}^{\prime}+1}^{-1}(Z) \backslash S\left(\mathscr{Z}^{\mathscr{F}^{\prime}}\right)
$$

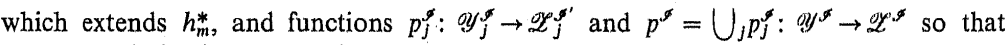
$h^{*}(\partial Y)=\partial\left(p^{*}(Y)\right)$ for $Y \in \mathscr{Y}$. $^{*}$

Finally, we can define $h_{m+1}^{*}$ to be $h_{m}^{*}$ on the union (4.7), and $h_{g}^{*}$ on every $Y_{g} \in y^{m}$ $p_{j}^{m+1}$ can be defined by $p_{j}^{m+1}=\bigcup_{\mathscr{F}} p_{j}^{\xi}$ where $\mathscr{I}$ runs over all $(m+1)$-element sets $\mathscr{I}=\left\{i_{1}, \ldots, i_{m+1}\right\}$ of integers. This completes the inductive definition of $h_{m}^{*}$.

It is easy to see that now we can define a homeomorphism

$$
\hbar^{*}=\bigcup_{m=1}^{\infty} h_{m}^{*}: X \backslash \tilde{Y} \rightarrow \bar{X} \backslash \tilde{Z} .
$$

Then we can uniquely extend $\bar{h}^{*}$ to $h^{*}: X \rightarrow \bar{X}$ by $h^{*}\left(\widetilde{Y}_{\left(i_{1}, i_{2}, \ldots\right)}\right)=\tilde{Z}_{\left(i_{1}, i_{2}, \ldots\right)}$ where $\left\{i_{1}^{\prime}, i_{2}^{\prime}, \ldots\right\}$ is the uniquely defined sequence of integers such that for every $m$, $p^{m}\left(Y_{i_{1}, \ldots, i_{m}+1}\right)=Z_{i_{1}^{\prime}, \ldots, i_{m+1}^{\prime}}$.
Corollary (4.7). Let $X \in X\left(L^{n} \# N, \mathscr{M}\right)$ and let $X^{\prime} \in X\left(L^{n} \# N^{\prime}, \mathscr{M}\right)$, where $N$ and $N^{\prime}$ are connected sums of a finite number of manifolds homeomorphic to elements of $.1^{n}$. Then $X$ and $X^{\prime}$ are homeomorphic.

Proof. We can construct systems $\left\{\mathscr{M}^{n}, L_{i}, x_{i, i+1}, \Omega_{i}\right\}$ and $\left\{\mathscr{M}^{n}, \bar{L}_{i}, \bar{\alpha}_{i, i+1}, \bar{\Omega}_{i}\right\}$ with $L_{1}=\bar{L}_{1}=L^{n}$ such that $L_{i^{\prime}} \approx L^{n} \# N$ and $\bar{L}_{i^{\prime \prime}} \approx L^{n} \# N^{\prime}$ for some integers $i^{\prime}, i^{\prime \prime}$, so that both systems define a space from $X\left(L^{n}, \mathscr{M}^{n}\right)$ but after restricting the first of them to $L_{i}$ with $i>i^{\prime}$ and the second to $\bar{L}_{i}$ with $i>i^{\prime \prime}$ they define spaces from $X\left(L^{n} \# N, \mathscr{M}^{n}\right)$ and $X\left(L^{n} \# N^{\prime}, \mathscr{M}^{n}\right)$ respectively.

5. Construction of $X\left(L^{n}, \mathscr{M}^{n}\right)$. We now know that for every $L^{n}$ and $\mathscr{M}^{n}$ there exists at most one space $X \in X\left(L^{n}, \mathscr{M}^{n}\right)$. We will denote it simply $X\left(L^{n}, \mathscr{M}^{n}\right)$. In this section we will construct a space $X\left(L^{n}, \mathscr{M}^{n}\right)$ for any $L^{n}$ and $\mathscr{M}^{n}$, thus proving that for every $L^{n}$ and $\mathscr{M}^{n}$ there exists precisely one space $X\left(L^{n}, \mathscr{M}^{n}\right)$. We assume the following:

Data (5.1). If $\mathscr{M}^{n}$ is infinite, then $\left\{j_{1}, j_{2} \ldots\right\}$ is an infinite sequence of integers in which every integer appears infinitely many times. If $\mathscr{M}^{n}$ is finite, then $\left\{j_{1}, j_{2}, \ldots\right\}$ is an infinite sequence of integers $\leqslant s$, where $s$ is the cardinality of $\mathscr{M}^{n}$, in which every such integer appears infinitely many times.

For any $L^{n}$ and $\mathscr{M}^{n}=\left\{M_{i}\right\}_{i \in N}$ we will construct a system $\left\{\mathscr{M}^{n}, L_{i}, \alpha_{i, i+1}, \Omega_{i}\right\}$ defining a space $X=X\left(L^{n}, \mathscr{M}^{n}\right)$ which moreover satisfies the following condition:

$\mathrm{A}\left(\left\{j_{1}, j_{2}, \ldots\right\}\right)$ : For every $k \in N$ and every $Y \in \Omega_{k}$ we have $\alpha_{k, k+1}^{-1}(Y) \approx M_{j_{k}} \backslash D^{n}$.

Moreover, for every $\varepsilon>0$ there exists $k_{0}$ such that for every $k>k_{0}$ we have $\operatorname{diam}\left(\alpha_{k}^{-1}(Y)\right)<\varepsilon$ for every $Y \in \Omega_{k}$ and for every $x \in X\left(L^{n}, \mathscr{M}^{n}\right)$ there exists $Y \in \Omega_{k}$ with $\operatorname{dist}\left(x, \alpha_{k}^{-1}(Y)\right)<\varepsilon$.

The construction is inductive: let $\left\{\varepsilon_{1}, \varepsilon_{2}, \ldots\right\}$ be a sequence of positive numbers converging to 0 . We take $L_{1}=L^{n}$ and for $\Omega_{1}$ we take any finite collection of disjoint, bicollared $n$-cells in $L_{1}$ having diameters $<\varepsilon_{1}$ and such that for every $x \in L_{1}$ there exists $Y \in \Omega_{1}$ with $\operatorname{dist}(x, Y)<\varepsilon_{1}$. Then assume inductively that we have defined spaces $L_{1}, \ldots, L_{k}$, maps $\alpha_{i, i+1}$ for $i<k$ and families $\Omega_{i}$ for $i \leqslant k$ so that axioms (1), (2) are satisfied for $i<k$, axioms (3), (4) are satisfied for $i<k$ and axiom (5) is satisfied for $i, j \leqslant k$. Moreover, we require that $\operatorname{diam}(Y) \leqslant \varepsilon_{i}$ for $i \leqslant k$ and $Y \in \Omega_{i}$, for any $x \in L_{i}$ there exists $Y \in \Omega_{i}$ with $\operatorname{dist}(x, Y)<\varepsilon_{i}$, and $\alpha_{i, i+1}^{-1}(Y) \approx M_{j_{i}} \backslash D^{n}$ for every $i<k$ and $Y \in \Omega_{i}$.

Then we construct $L_{k+1}$ as follows: we remove the interior of every $Y \in \Omega_{k}$ from $L_{k}$, and instead of it we glue in a copy of the manifold $M_{j_{k}} \backslash D^{n}$, identifying its boundary with $\partial Y$. We choose a metric on $L_{k+1}$ to coincide with the metric of $L_{k}$ on $L_{k} \cap L_{k+1}$, and on the attached manifolds we choose it so that each attached copy of $M_{j_{k}} \backslash D^{n}$ has diameter $<\varepsilon_{k}$. Then we define $\alpha_{k, k+1}$ to be identity on $L_{k} \cap L_{k+1}$; on every attached copy of $M_{j_{k}} \backslash D^{n}$ we let $\alpha_{k, k+1}$ be any map onto the corresponding $Y \in \Omega_{k}$ which extends identity on $\partial Y$ and for which $\alpha_{k, k+1} \mid \alpha_{k, k+1}^{-1}(\partial Y): \alpha_{k, k+1}^{-1}(\partial Y) \rightarrow \partial Y$ is a homeomorphism. The set $Z_{k+1}=\bigcup_{i \leqslant k} \alpha_{i, k+1}^{-1}\left(\bigcup\left\{\partial Y: Y \in \Omega_{i}\right\}\right)$ is closed and nowhere dense in $L_{k+1}$. Therefore we can define $\Omega_{k+1}$ to be any finite family of disjoint, bicollared $n$-cells in $L_{k+1}$ such that for any $Y \in \Omega_{k+1}$ we have $Y \cap Z_{k+1}=\varnothing, \operatorname{diam}(Y)<\varepsilon_{k+1}$, and for any $x \in L_{k+1}$ there exists $Y \in \Omega_{k+1}$ with $\operatorname{dist}(x, Y)<\varepsilon_{k+1}$. It is easy to see that then the inductive hypothesis is 
met with $k$ replaced by $k+1$. If we perform all the steps of the induction, we get a system $\left\{\mathscr{M}^{n}, L_{i}, \alpha_{i, i+1}, \Omega_{i}\right\}$ which satisfies axioms (1)-(5). Axioms (6) and (7) are satisfied because of the way in which the families $\Omega_{i}$ depend on $\left\{\varepsilon_{i}\right\}_{i \in N}$. Finally, the system satisfies $\mathrm{A}\left(\left\{i_{1}, i_{2}, \ldots\right\}\right)$, so axiom (8) is satisfied because of the properties of the sequence $\left\{i_{1}, i_{2}, \ldots\right\}$.

6. Embeddings of $X\left(L^{n}, \mathscr{M}^{n}\right)$ into manifolds. Let $X=X\left(L^{n}, \mathscr{M}^{n}\right)$, let $\left\{\mathscr{M}^{n}, L_{l}, \alpha_{i, i+1}, \Omega_{i}\right\}$ be a defining system for $X$, and let $P$ be a manifold (possibly with boundary) of dimension $k>n$. We will use all the notation introduced in Section 4. In particular, we have $\mathscr{M}^{n}=\left\{M_{j}\right\}_{, \in N}, \Omega_{1}=\mathscr{Y}^{\varnothing}, \Omega_{1}^{\prime}\left(M_{j}\right)=\mathscr{Y}_{j}^{\varnothing}$ and we have families $\mathscr{Y}^{\mathscr{\mathscr { G }}}=\mathscr{Y}_{1} \cup \mathscr{Y}_{2} \cup \ldots$ for $\mathscr{I}=\left\{i_{1}, \ldots, i_{m}\right\}$. We assume the following:

Data (6.1). There exists a bicollared topological embedding $e_{L}: L^{n} \rightarrow P$ with $e_{L}\left(\partial L^{n}\right)=e_{L}\left(L^{n}\right) \cap \partial P$, and for every $M \in \mathscr{M}^{n}$ there exists a bicollared embedding $e_{M}: M \backslash D^{n} \rightarrow D^{k}$ (where $D^{n} \subset M$ ) such that $e_{M}\left(\partial D^{n}\right)=e_{M}\left(M \backslash D^{n}\right) \cap \partial D^{k}$ and $e_{M}\left(\partial D^{n}\right)$ is a standard $(n-1)$-sphere $S^{n-1} \subset S^{k-1}=\partial D^{k}$.

Assuming (6.1) we will construct an embedding of $X$ into $P$. First, having $e_{L}: L \rightarrow P$ and any fixed sequence $\delta_{1}>\delta_{2}>\ldots$ of positive numbers converging to 0 , we construct inductively a sequence of embeddings

$$
e_{L}^{m}: L_{m} \rightarrow P
$$

where $L_{0}=L$, and

$$
\begin{aligned}
L_{m}= & \left(L \backslash S\left(\mathscr{Y}^{Ø}\right)\right) \cup \bigcup_{i_{1} \in N}\left(\alpha_{1, k_{1}+1}^{-1}\left(Y_{i_{1}}\right) \backslash S\left(\mathscr{Y}^{\left(i_{1}\right\}}\right)\right) \cup \ldots \\
& \ldots \cup \cup \bigcup_{i_{1}, \ldots, i_{m} \in N}\left(\alpha_{k_{m-1}+1, k_{m}+1}^{-1}\left(Y_{i_{1}, \ldots, i_{m}}\right) \backslash S\left(\mathscr{Y}^{\left(i_{1}, \ldots, i_{m}\right.}\right)\right) \cup \bigcup_{i_{1}, \ldots, i_{m} \in N} S\left(\mathscr{Y}^{\left(i_{1}, \ldots, i_{m}\right\}}\right),
\end{aligned}
$$

where $k_{m}=k_{m}\left(i_{1}, \ldots, i_{m}\right)$ in every summand.

We claim that these embeddings have the following properties:

$\mathrm{a}(m)$ : For every $m$ the maps $e_{L}^{m}$ and $e_{L}^{m-1}$ coincide on the space

$$
\begin{aligned}
& L_{m-1} \cap L_{m}=\left(L^{n} \backslash S\left(\mathscr{Y}^{\varnothing}\right)\right) \cup \bigcup_{i_{1} \in N}\left(\alpha_{1, k_{1}+1}^{-1}\left(Y_{i_{1}}\right) \backslash S\left(\mathscr{Y}^{\left(i_{1}\right\rfloor}\right)\right) \cup \ldots \\
& \ldots \cup \bigcup_{i_{1}, \ldots, i_{m-1} \in N}\left(\alpha_{k_{m-2}+1, k_{m-1}+1}^{-1}\left(Y_{i_{1}, \ldots, i_{m-1}}\right) \backslash S\left(\mathscr{Y}^{\left(i_{1}, \ldots, i_{m-1}\right)}\right)\right)
\end{aligned}
$$

$\mathrm{b}(m)$ : For every $m$ and every set $\mathscr{I}=\left\{i_{1}, \ldots, i_{m}\right\}$ consisting of $m$ integers there is a bicollared embedding

$$
e^{\mathscr{F}}: D^{k} \rightarrow P
$$

such that $e^{\left\{i_{1}, \ldots, i_{m-1}\right\}}\left(D^{k}\right) \supset e^{\left\{i_{1}, \ldots, i_{m}\right\}}\left(D^{k}\right)$ and $e^{s}\left(D^{k}\right) \cap e^{s}\left(D_{k}\right)=\varnothing$ for any two $m$-element sets $\mathscr{I} \neq \mathscr{I}^{\prime}$; moreover:

$\mathrm{c}(m): e^{\mathcal{J}}\left(D^{k}\right) \cap e_{L}^{m}\left(L_{m}\right)=e_{L}^{m}\left(\alpha_{k_{m-1}+1, k_{m}+1}^{-1}\left(Y_{i_{1}, \ldots, i_{m}}\right)\right)$ is a topological, bicollared submanifold of $e^{\mathscr{J}}\left(D^{k}\right)$ for $\mathscr{I}=\left\{i_{1}, \ldots, i_{m}\right\}$ and $\left(e^{\xi}\right)^{-1}\left(e^{\xi}\left(\partial D^{k}\right) \cap e_{L}^{m}\left(L_{m}\right)\right)=S^{n-1} \subset S^{k-1}$ is the standard $(n-1)$-sphere in $\partial D^{k}=S^{k-1}$

$\mathrm{d}(m): \operatorname{diam}\left(e^{\mathscr{J}}\left(D^{k}\right)\right)<\delta_{m}$ for $\mathscr{I}=\left\{i_{1}, \ldots, i_{m}\right\}$.

$\mathrm{e}(m): \operatorname{diam}\left(e_{L}^{m}\left(Y_{i_{1}, \ldots, i_{m+1}}\right)\right)<\delta_{m+1}$ for every $Y_{i_{1}, \ldots, i_{m+1}} \in \mathscr{Y}^{\left\{i_{1}, \ldots, i_{m}\right\}}$.
Let us take the map $e_{L}: L^{n} \rightarrow P$. Using Bing's shrinking criterion described for example in [F], p. 417, Lemma (7.1), we can find a homeomorphism $\xi: L^{n} \rightarrow L^{n}$ such that the embedding $e_{L}^{0}=e_{L} \xi: L^{n} \rightarrow P$ has the following property: $\left\{e_{L}^{0}\left(Y_{i_{1}}\right): Y_{i_{1}} \in \mathscr{Y}^{ø}\right\}$ is a null family with all elements of diameter $<\delta_{1}$. We start induction with this map.

Assume now that we have constructed $e_{L}^{m}$ satisfying a $(m)-\mathrm{e}(m)$. Let $\mathscr{I}=$

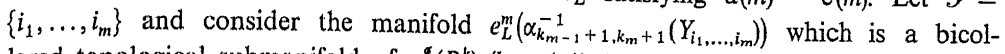
lared topological submanifold of $e^{\xi}\left(D^{k}\right)$ (by $\left.\mathrm{c}(m)\right)$. Using e $(m)$ we can easily produce embeddings $e^{\left\{i_{1}, \ldots, i_{m+1}\right)}: D^{k} \rightarrow P$ for every $i_{m+1} \in N$ so that $\mathrm{b}(m+1)$ and $\mathrm{d}(m+1)$ are satisfied. Then let $\eta: \alpha_{k_{m+1}, k_{m+1}+1}^{-1}\left(Y_{i_{1}, \ldots, i_{m+1}}\right) \rightarrow M_{j_{m+1}}$ be a homeomorphism, where $j_{m+1}=j_{m+1}\left(i_{1}, \ldots, i_{m+1}\right)$. We define an embedding

$$
f_{s, l_{m+1}}=e^{\left(l_{1}, \ldots, i_{m+1}\right)} \circ \eta: \alpha_{k_{m}+1, k_{m+1}+1}^{-1}\left(Y_{i_{1}, \ldots, i_{m+1}}\right) \rightarrow e^{\left\{i_{1}, \ldots, i_{m+1}\right\}}\left(D^{k}\right) .
$$

The map $f_{\mathscr{s}, i_{m+1}}$ extends $e_{L}^{m} \mid L_{m+1} \cap L_{m}$ if $\eta$ chosen properly on the boundary, but we cannot put $e_{L}^{m+1}=f_{\mathscr{s}, l_{m+1}}$, because $\mathrm{e}(m)$ would not be satisfied. But again we can compose $f_{s, i_{m+1}}$ with an automorphism $\xi$ of $\alpha_{k_{m}+1, k_{m+1}+1}^{-1}\left(Y_{i_{1}, \ldots, i_{m+1}}\right)$ obtained by Bing's shrinking criterion so that if we put $e_{L}^{m+1} \mid \alpha_{k_{m}+1, k_{m+1}+1}^{-1}\left(Y_{i_{1}, \ldots, i_{m+1}}\right)=f_{\mathcal{S}, i_{m+1}} \circ \xi$ then both $\mathrm{e}(m+1)$ and $\mathrm{c}(m+1)$ are satisfied.

So we have defined $e_{L}^{m+1}$ as equal to $e_{L}^{m}$ on $L_{m} \cap L_{m+1}$ and to $f_{s, i_{m+1}} \circ \xi$ on $\alpha_{k_{m}+1, k_{m+1}+1}^{-1}\left(Y_{i_{1}, \ldots, i_{m+1}}\right)$. Now, notice that $L_{m} \cap L_{m+1}$ is the union of the first $m+1$ summands of (4.5). This implies that we can define an embedding

$$
e_{X \backslash Y}: X \backslash \tilde{Y} \rightarrow P
$$

by $e_{X \backslash Y}\left|L_{m} \cap L_{m+1}=e_{L}^{m}\right| L_{m} \cap L_{m+1}$. This is well defined because $X \backslash \tilde{Y}=\bigcup_{m}\left(L_{m} \cap L_{m+1}\right)$ by (4.5). Now, we extend $e_{X \backslash Y}$ to a map $e_{X}: X \rightarrow P$ putting

$$
e_{X}\left(Y_{\left\{i_{1}, i_{2}, \ldots\right\}}\right)=\bigcap_{m=1}^{\infty} e^{\left\{i_{1}, \ldots, i_{m}\right\}}\left(D^{k}\right) .
$$

It can easily be seen from $\mathrm{a}(m)-\mathrm{e}(m)$ that $e_{X}$ is an embedding.

7. Defining systems satisfying a special condition. Let $X=X\left(L^{n}, \mathscr{M}^{n}\right)$ for some $L^{n}$ and $\mathscr{M}^{n}$ and let $\left\{\mathscr{M}^{n}, L_{i}, \alpha_{i, i+1}, \Omega_{i}\right\}$ be a defining system for $X$. Of course we have much freedom in choosing a defining system. Let $p_{1}, \ldots, p_{m} \in X$ be any finite sequence of distinct points in $X$. We are interested whether $\left\{\mathscr{M}^{n}, L_{i}, \alpha_{i, i+1}, \Omega_{i}\right\}$ satisfies the following condition:

$\mathbf{B}\left(p_{1}, \ldots, p_{m}\right)$ : There exists an increasing sequence $k_{1}, k_{2}, \ldots$ of integers, a manifold $M \in \mathscr{M} \|^{n}$, and a family of $n$-cells $\left\{Y_{1}^{j}, Y_{2}^{j}, \ldots\right\}$ for every $j \leqslant m$ such that $Y_{i}^{j} \in \Omega_{k_{i}}$, $\left.\alpha_{k_{i}, k_{i}+1}^{-1}(Y i) \approx M \backslash D^{n}, \alpha_{k_{1}, k_{i}+1}(Y i+1) \subset Y\right\}$ for any $i \in N$, and $p_{j}=\bigcap_{i=1}^{\infty} \alpha_{k_{i}}^{-1}\left(Y_{i}^{i}\right)$.

Lemma (7.1). For every $L^{n}$ and $\mathscr{M}^{n}$ and any points $p_{1}, \ldots, p_{m} \in X=X\left(L^{n}, \mathscr{M}^{n}\right)$, there exists a system $\left\{\mathscr{M}^{n}, L_{i}, \alpha_{i, i+1}, \Omega_{i}\right\}$ defining $X$ which satisfies condition $\mathrm{B}\left(p_{1}, \ldots, p_{m}\right)$.

Proof. By the uniqueness theorem (4.6) and by the construction described in Section 5 , we can find a system $\left\{\mathscr{M}^{n}, L_{i}, \alpha_{i, l+1}, \bar{\Omega}_{i}\right\}$ defining $X$ and satisfying condition $\mathrm{A}\left(\left\{j_{1}, j_{2}, \ldots\right\}\right)$ for some sequence $\left\{j_{1}, j_{2}, \ldots\right\}$ which satisfies (5.1). Note that in $\mathrm{A}\left(\left\{j_{1}, j_{2}, \ldots\right\}\right)$ we assume our family $\mathscr{M}^{n}$ to be indexed: $\mathscr{M}^{n}=\left\{M_{i}\right\}_{i \in N}$. For every $j \leqslant m$ we can choose a family $\{U\}\}_{i \in N}$ of open subsets of $X$ such that $U_{1}^{j} \supset U_{2}^{j} \supset \ldots$, 
$U_{1}^{j} \cap U_{1}^{j^{\prime}}=\varnothing$ for $j \neq j^{\prime}$ and $p_{j}=\bigcap_{i=1}^{\alpha} U_{i}^{j}$. Moreover, we can assume that $V_{k, i}^{j}=\alpha_{k}\left(U_{i}^{\prime}\right)$ is an open $\bar{\Omega}_{k}^{\prime}$-saturated subset of $L_{k}$ for any $k \geqslant i$, i.e. for every $Y \in \bar{\Omega}_{k}^{\prime}$ either $Y \cap V_{k, i}^{j}=\varnothing$ or $Y \subset V_{k, i}^{\dot{R}}$.

We will modify inductively the families $\bar{\Omega}_{i}$ in the system $\left\{\mathscr{M}^{n}, L_{i}, \alpha_{i, i+1}, \bar{\Omega}_{i}\right\}$ so as to get a new system $\left\{\mathscr{M}^{n}, L_{i}, \alpha_{i, i+1}, \Omega_{i}\right\}$ defining $X$ and satisfying $\mathrm{B}\left(p_{1}, \ldots, p_{m}\right)$.

By $\mathrm{A}\left(\left\{j_{1}, j_{2}, \ldots\right\}\right)$ we can find an integer $k_{1}>1$ such that for $j \leqslant m$ there are $n$-cells $Y_{j} \in \bar{\Omega}_{k_{1}}$ such that $\alpha_{k_{1}}^{-1}\left(Y_{j}\right) \subset U_{1}^{j}$ and $M_{j_{k_{1}}}=M$, and consequently $\alpha_{k_{1}, k_{1}+1}^{-1}\left(Y_{j}\right) \approx M \backslash D^{n}$.

Moreover, if $\alpha_{k_{1}}\left(p_{j}\right) \in Y$ for some $Y \in \widetilde{\Omega}_{k_{1}}$ then we can assume that $X_{j}=Y$.

Now for $j \leqslant m$ we will define $n$-cells $Y_{1}^{J} \subset V_{k_{1}, 1}^{\prime}=\alpha_{k_{1}}\left(U_{1}^{J}\right)$ with the following properties: $\operatorname{Int}\left(Y_{1}^{j}\right) \supset Y_{j} \cup\left\{\alpha_{k_{1}}\left(p_{j}\right)\right\}, \partial Y_{1}^{j}$ does not intersect any element of ${\overline{\Omega_{k}}}_{k_{1}}^{\prime}$ and $Y_{1}^{j}$ does not intersect any element of the family

$$
\left\{\alpha_{k, k_{1}}^{-1}(T): k \leqslant k_{1} \text { and } T \in \bar{\Omega}_{k}\right\} \backslash\left\{Y_{j}\right\} .
$$

We construct the sets $Y_{1}^{j}$ as follows: we take a decomposition $\pi: L_{k_{1}} \rightarrow L_{k_{1}} / \bar{\Omega}_{k_{1}}^{\prime}=\tilde{L}_{k_{1}}$. By a theorem of Bing (Theorem (7.2) in [F]) $\tilde{L}_{k_{1}}$ is homeomorphic to $L_{k_{1}}$ and the non-degenerate elements of this decomposition form a dense countable subset in $\tilde{L}_{k_{1}}$. Then for every $j \leqslant m$ we find a bicollared $n$-cell $\tilde{Y} \subset \tilde{L}_{k_{1}}$ such that Int $\tilde{Y}$ contains $\pi\left(\alpha_{k_{1}}\left(p_{1}\right)\right) \cup \pi\left(Y_{j}\right)$. Because of our choice of $Y_{j}$ we can assume that

$$
\tilde{Y} \cap \pi\left(\left\{\alpha_{k, k_{1}}^{-1}(T): k \leqslant k_{1} \text { and } T \in \widetilde{\Omega}_{k}\right\}\right)=\pi\left(Y_{\jmath}\right) \text {. }
$$

By [B-P], p. 140, Theorem (7.2), we can assume that $\partial \tilde{Y} \cap \pi\left(\widetilde{\Omega}_{k_{1}}^{\prime}\right)=\varnothing$. Finally, we can choose $\tilde{Y}$ so small that $\tilde{Y} \subset \pi\left(V_{k, 1}^{j}\right)$. Then we define $Y_{1}^{j}=\pi^{-1}(\tilde{Y})$. It is easy to see that $Y_{1}^{j}$ so constructed has all required properties. To complete the first step of induction we put $\Omega_{i}=\bar{\Omega}_{i}$ for $i \neq k_{1}$ and $\Omega_{k_{1}}=\bar{\Omega}_{k_{1}} \cup\left\{Y_{1}^{1}, \ldots, Y_{1}^{m}\right\} \backslash\left\{Y_{1}, \ldots, Y_{m}\right\}$.

Now assume that we have integers $k_{1}<\ldots<k_{s}$ and a system $\left\{\mathscr{M}^{n}, L_{i}, \alpha_{i, i+1}, \bar{\Omega}_{i}\right\}$ defining $X$ such that for every $j \leqslant m$ there exists a family of $n$-cells $\left\{Y_{1}^{j}, \ldots, Y_{s}^{j}\right\}$ with $Y_{i}^{j} \in \bar{\Omega}_{k_{i}}, \alpha_{k_{i}, k_{i}+1}^{-1}\left(Y_{i}^{j}\right) \approx M \backslash D^{n}$ for $i \leqslant s . \alpha_{k_{i}, k_{i+1}}\left(Y_{i+1}^{j}\right) \in V_{k_{i}, i+1}^{j} \cap Y_{i}^{j}$ for $i<s$ and $Y_{s}^{j} \ni \alpha_{k_{x}}\left(p_{j}\right)$ (the definition of $V_{k, i}^{j}$ remains unchanged). By $\mathrm{A}\left(\left\{j_{1}, j_{2}, \ldots\right\}\right)$ we can find an integer $k_{s+1}>k_{s}$ such that for $j \leqslant m$ there are $n$-cells $Y_{j} \in \bar{\Omega}_{k_{x+1}}$ such that $\alpha_{k_{n+1}}^{-1}\left(Y_{j}\right)$ $\subset U_{s+1}^{j} \cap \operatorname{Int}\left(\alpha_{k_{s}}^{-1}\left(Y_{s}^{j}\right)\right)$ and that $M_{j k_{k+1}}=M$.

Moreover, we can assume; that if $\alpha_{k_{x+1}}\left(p_{j}\right) \in Y$ for some $Y \in \bar{\Omega}_{k_{x+1}}$ then $Y_{j}=Y$.

Now we define $n$-cells $Y_{s+1}^{j} \subset V_{k_{x+1}, s+1}^{j} \cap \alpha_{k_{s}, k_{x+1}}^{-21}\left(Y_{s}^{j}\right)$ in the same way as for $Y_{1}^{j}$. We complete the induction by putting $\Omega_{l}=\widetilde{\Omega}_{l}$ for $i \neq k_{x+1}$ and $\Omega_{k_{*+1}}=\bar{\Omega}_{k_{x+1}}$ $\cup\left\{Y_{s+1}^{1}, \ldots, Y_{s+1}^{m}\right\} \backslash\left\{Y_{1}, \ldots, Y_{m}\right\}$.

Every step of this induction changes only one family $\Omega_{i}$. This implies that performing all the steps we get a system $\left\{\mathscr{M}^{n}, L_{l}, \alpha_{i, i+1}, \Omega_{l}\right\}$ which still satisfies axioms (1)-(8) and $\mathbf{A}\left(\left\{j_{1}, j_{2}, \ldots\right\}\right)$ and additionally it satisfies $\mathbf{B}\left(p_{1}, \ldots, p_{m}\right)$.

8. The $m$-homogeneity of $X\left(L^{n}, \mathscr{M}^{n}\right)$. A space $X$ is said to be m-homogeneous if for any two $\dot{m}$-element collections of distinct points $\left\{p_{1}, \ldots, p_{m}\right\}$ and $\left\{\bar{p}_{1}, \ldots, \bar{p}_{m}\right\}$ in $X$ there exists a homeomorphism $h: X \rightarrow X$ such that $h\left(p_{i}\right)=\bar{p}_{i}$ for $i \leqslant m$. We prove the following:
THeOREM (8.1). For every closed manifold $L^{n}$, every family $\mathscr{M}^{n}$ and every positive integer $m$ the space $X=X\left(L^{n}, \mathscr{M}^{n}\right)$ is m-homogeneous.

Proof. Let $\left\{p_{1}, \ldots, p_{m}\right\}$ and $\left\{\bar{p}_{1}, \ldots, \bar{p}_{m}\right\}$ be two collections of points. Without loss of generality we can assume that the collections are disjoint. By (7.1) there exists a system $\left\{\mathscr{M}^{n}, L_{i}, \alpha_{i, i+1}, \Omega_{i}\right\}$ defining $X$ and satisfying $\mathrm{B}\left(p_{1}, \ldots, p_{m}, \bar{p}_{1}, \ldots, \bar{p}_{m}\right)$. Then of course the conditions $\mathrm{B}\left(p_{1}, \ldots, p_{m}\right)$ and $B\left(\bar{p}_{1}, \ldots, \bar{p}_{m}\right)$ are also satisfied, and moreover an increasing sequence $k_{1}<k_{2}<\ldots$ determined by these two conditions is the same.

This implies that for every $j \leqslant \mathrm{~m}$ there are families $\left\{Y_{1}^{j}, Y_{2}^{j}, \ldots\right\}$ and $\left\{\bar{Y}_{1}^{1}, \bar{Y}_{2}^{j}, \ldots\right\}$, with $Y_{i}^{j}, Y_{l}^{j} \in \Omega_{k_{i},}, \alpha_{k_{1}, k_{i}+1}^{m 1_{1}}\left(Y_{i}^{j}\right) \approx \alpha_{k_{1}, k_{i}+1}^{-1}\left(\bar{Y}_{i}^{j}\right) \approx M \backslash D^{n}$ for some fixed $M \in \mathscr{M}^{n}, \alpha_{k_{i}, k_{i+1}}\left(Y_{i+1}^{j}\right)$ $\subset Y_{i}^{j}, \alpha_{k_{1}, k_{l+1}}\left(\bar{Y}_{i+1}^{j}\right) \subset Y_{i}^{\prime}$ and $p_{j}=\bigcap_{i=1}^{\infty} \alpha_{k_{i}}^{-1}\left(Y_{i}^{j}\right), \bar{p}_{j}=\bigcap_{i=1}^{\infty} \alpha_{k_{i}}^{-1}\left(\bar{Y}_{i}^{j}\right)$.

We construct inductively a homeomorphism $h: X \rightarrow X$ with $h\left(p_{j}\right)=\bar{p}_{j}, j \leqslant m$. First we take the spaces

Obviously

$$
X_{1}=X \backslash \alpha_{k_{1}}^{-1}\left(\bigcup_{j=1}^{m} Y_{1}^{j}\right) \quad \text { and } \quad \bar{X}_{1}=X \backslash \alpha_{k_{1}}^{-1}\left(\bigcup_{j=1}^{m} \bar{Y}_{1}^{j}\right)
$$

$$
X_{1} \in X\left(L_{k_{1}} \backslash \bigcup_{j=1}^{m} \operatorname{Int} Y_{1}^{j}, \mathscr{M}\right) \quad \text { and } \quad \bar{X}_{1} \in X\left(L_{k_{1}} \backslash \bigcup_{j=1}^{m} \operatorname{Int} \bar{Y}_{1}^{j}, \mathscr{M}\right)
$$

so by Theorem (4.6) there is a homeomorphism $h_{1}: X_{1} \rightarrow \bar{X}_{1}$ such that

$$
h_{1}\left(\alpha_{k_{1}}^{m-1}\left(\partial Y_{1}^{j}\right)\right)=\alpha_{k_{1}}^{-1}\left(\partial \bar{Y}_{1}^{j}\right) \quad \text { for } j \leqslant m \text {. }
$$

Then we set

$$
X_{s}=X \backslash \bigcup_{j=1}^{m} \alpha_{k_{s}}^{\alpha_{n}-1}\left(\operatorname{Int} Y_{s}^{j}\right) \quad \text { and } \quad \bar{X}_{s}=X \backslash \bigcup_{j=1}^{m} \alpha_{k_{s}}^{-1}\left(\operatorname{Int} \bar{Y}_{s}^{j}\right)
$$

and assume inductively that we have a homeomorphism $h_{s}: X_{s} \rightarrow \bar{X}_{s}$ such that

$$
h_{s}\left(\alpha_{k_{s}}^{-1}\left(\partial Y_{s}^{j}\right)\right)=\alpha_{k_{s}}^{-1}\left(\partial \bar{Y}_{s}^{j}\right) \quad \text { for } j \leqslant s
$$

For every $j \leqslant m$ we find a homeomorphism

$$
h_{s+1}^{J}: \alpha_{k_{u}}^{-1}\left(Y_{s}^{J}\right) \backslash \alpha_{k_{w+1}}^{-1}\left(\operatorname{Int} Y_{s+1}^{J}\right) \rightarrow \alpha_{k_{x}}^{-1}\left(\bar{Y}_{s}^{J}\right) \backslash \alpha_{k_{s+1}}^{-1}\left(\operatorname{Int} \bar{Y}_{s+1}^{J}\right)
$$

which extends $h_{s}$, i.e. agrees with $h_{s}$ on $\alpha_{k_{x}}^{-1}\left(\partial Y_{s}^{j}\right)$. Indeed, the spaces $\alpha_{k_{x}}^{-1}\left(Y_{s}^{j}\right) \backslash \alpha_{k_{s}+1}^{-1}\left(\right.$ Int $\left.Y_{s+1}^{j}\right)$ and $\alpha_{k_{n}}^{\prime 1}\left(Y_{s}^{\prime}\right) \backslash \alpha_{k_{*}+1}^{n}\left(\right.$ Int $\left.Y_{s+1}^{\prime}\right)$ belong to the classes $X\left(\left(M \backslash D_{1}^{n} \backslash D_{2}^{n}\right) \# N, \mathscr{M}\right)$ and $X\left(\left(M \backslash D_{1}^{\prime \prime} \backslash D_{2}^{\prime \prime}\right) \not \#, \mathscr{N}, \mathscr{H}\right)$ respectively where $D_{1}^{\prime \prime}$ and $D_{2}^{n}$ are two disjoint $n$-disks in $M$, and $N$ and $N$ are both connected sums of a finite number of manifolds from $\mathscr{M}$ (even though not necessarily $N=N$ ). So by Corollary (4.7) the required homeomorphism $h_{s+1}^{J}$ exists. Then we define $h_{s+1}: X_{s+1} \rightarrow \bar{X}_{s+1}$ by $h_{s+1} \mid X_{s}=h_{s}$ and $h_{s+1} \mid \alpha_{k_{s}}^{\prime \prime 1}\left(Y_{s}^{J}\right) \backslash \alpha_{k_{*+1}+1}^{* 1}\left(\operatorname{Int} Y_{s+1}^{\prime}\right)=h_{s+1}^{J}$ for $j \leqslant m$.

Finally, we define $h: X \rightarrow X$ by $h \mid X_{s}=h_{s}$ and $h\left(p_{j}\right)=\bar{p}_{j}$ for $j \leqslant m$.

9. The spaces $X\left(L^{n}, \mathscr{H}^{n}\right)$ as fixed-point sets of topological Lie group actions on manifolds. In this section we show how the spaces $X\left(L^{n}, \not \mathscr{L}^{n}\right)$ can naturally appear as fixed-point sets of topological actions of compact Lie groups on manifolds. 
EXAmple (9.1). Let $\mathscr{M}^{3}$ be any finite or countable family of homology 3-spheres, and let $L^{3}=S^{3}$. We take $e_{L}: S^{3} \varsigma S^{4}$ to be the standard inclusion. By [F], Theorem (1.4), p. 367, for every $M \in \mathscr{M}^{3}$ there exsits a topological, bicollared (but not always smooth) embedding $e_{M}: \stackrel{M}{M} \backslash D^{3} \rightarrow D^{4}$ such that there exists an involution $i_{M}: D^{4} \rightarrow D^{4}$ with $\operatorname{Fix}\left(i_{M}\right)=e_{M}\left(M \backslash D^{3}\right)$. Now, applying the construction from Section 6 to the space $X=X\left(S^{3}, \mathscr{M}^{3}\right)$, we get an embedding

$$
e_{X}: X \rightarrow S^{4} \text {. }
$$

We have the standard orientation-reversing involution $i: S^{4} \rightarrow S^{4}$ with $\mathrm{Fix}(i)=S^{3}$, and for every $\mathscr{I}=\left\{i_{1}, \ldots, i_{m}\right\}$ we have an involution

$$
i_{\mathscr{F}}: e^{\prime \prime}\left(D^{4}\right) \rightarrow e^{\prime \prime}\left(D^{4}\right)
$$

with Fix $\left(i_{, \xi}\right)=e^{\xi}\left(D^{4}\right) \cap e_{L}^{m}\left(L_{m}\right)=e_{L}^{m}\left(\alpha_{k_{m-1}+1, k_{m}+1}^{-1}\left(Y_{l_{1}, \ldots . l_{m}}\right)\right)$ (see c $(m)$, Section 6). Moreover, we can assume that for any $\mathscr{I}$ we have

$$
i_{\mathscr{F}}\left|e^{\mathscr{F}}\left(\partial D^{4}\right)=i_{\left\{i_{1}, \ldots, i_{m-1}\right\}}\right| e^{\mathscr{F}}\left(D^{4}\right) \text {. }
$$

Of course $i_{s}$ is an involution induced by $i_{M_{J_{m}}}$, where $j_{m}=j_{m}\left(i_{1}, \ldots, i_{m}\right)$.

Now we can define an involution $i_{X}: S^{4} \rightarrow S^{4}$ by

$$
\begin{aligned}
& i_{X}=i \quad \text { on } \quad S^{4} \backslash \bigcup_{i_{1} \in N} e^{\left\{i_{1}\right\}}\left(D^{4}\right), \\
& i_{X}=i_{F} \quad \text { on } \quad e^{S}\left(D^{4}\right) \backslash \bigcup_{l_{m+1} \in N} e^{\left[i_{1}, \ldots, i_{m}, l_{m+1}\right)}\left(D^{4}\right),
\end{aligned}
$$

where $\mathscr{I}=\left\{i_{1}, \ldots, i_{m}\right\}$. Of course $e_{X}(X)=\operatorname{Fix}\left(i_{X}\right)$. The quotient space $S^{4} / i_{X}$ consists of a contractible, non-compact 4-manifold and a "compactifying" cohomology manifold $X$. As was discovered by Ancel and Siebenmann, for certain homology spheres $M$, $X\left(S^{3},\{M\}\right)$ compactifies a simply connected 4-manifold of Davis which covers a closed 4-dimensional manifold.

EXAMPLE (9.2). We take again $L^{3}=S^{3}$, and let $\mathscr{M}^{3}$ be a family of homology 3-spheres. Let $M \in \mathscr{M}^{3}$ and let $e_{M}: M \backslash D^{3} \rightarrow D^{5}$ be a smooth embedding with $e_{M}\left(\partial D^{3}\right) \subset \partial D^{5}$. By [M-Y] if every $M \in \mathscr{M}^{3}$ bounds a contractible manifold, then there is an action $\varphi_{m}$ of $S^{1}$ on $D^{5}$, standard on $\partial D^{5}$, and such that $e_{M}\left(M \backslash D^{\prime \prime}\right)=$ Fix $\left(\varphi_{M}\right)$. Using the same procedure as in (9.1) with involutions replaced by $S^{1}$-actions, we get an $S^{1}$-action $\varphi_{X}$ on $S^{5}$ with Fix $\left(\varphi_{X}\right)=e_{X}(X)$ where $e_{X}: X \rightarrow S^{5}$ is the embedding of the space $X=X\left(S^{3}, \mathscr{M}^{3}\right)$ described in Section 6 .

The two examples show the way of obtaining a great variety of topological Lie group actions on manifolds with fixed-point sets $X\left(L^{n}, \mathscr{M}^{n}\right)$ and orbit spaces quotients which are manifolds compactified by $X\left(L^{n}, \mathscr{M}^{n}\right)$.

10. The space $X\left(S^{3},\left\{H^{3}\right\}\right)$ as the orbit space of an action of an infinite 0-dimensional compact group. In [Ko] Kolmogorov gives an example of a 2-adic group acting effectively on a 1-dimensional locally connected continuum so that the orbit space is the 2-dimensional continuum of Pontryagin (see also [W]).
Here we give another similar example of an effective action of an infinite compact 0 -dimensional group on a locally connected continuum $P$ such that the orbit space is the cohomology manifold $X\left(S^{3},\left\{H^{3}\right\}\right)$. Here $H^{3}$ is a Poincare homology sphere (see $[\mathrm{K}-\mathrm{S}])$. First we construct $P$ : let $\varphi$ be an action of the binary icosahedral group $I^{*}$ on $S^{3}$ so that $S^{3} / I^{*}$ is a Poincare 3 -sphere $H^{3}$, and the projection onto the orbit space of this action $\pi_{p p}: S^{3} \rightarrow S^{3} / I^{*}=H^{3}$ is a 120 -fold covering. Let $D^{3} \subset H^{3}$ be a bicollared 3-cell. Then we take the space $R^{\prime}=S^{3} \backslash \pi_{\varphi}^{-1}\left(D^{3}\right)$ which is a 3-sphere with 120 holes. We identify all the components of $\partial R^{\prime}$ which consists of 120 copies of a 2-sphere, to one 2-sphere. We get a projection $\pi: R^{\prime} \rightarrow R$ onto a space $R$. $R$ admits an action $\varphi^{\prime}$ of $I^{*}$ with quotient map $\pi_{t p^{\prime}}: R \rightarrow H^{3} \backslash D^{\prime \prime}$ such that the following diagram commutes:

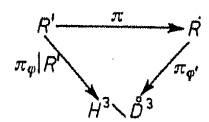

Let $\partial R=\pi\left(\partial R^{\prime}\right)$. Now we construct $P$ in the following way: we remove from $S^{3}$ the union of interiors of a dense, countable null family of bicollared 3-cells. We get a space $Z_{1}$ with "boundary" consisting of a countable family of 2-spheres. Then we attach to $Z_{1}$ a countable family $\left\{R_{i_{1}}\right\}_{i_{1} \in N}$ of copies of $R$ so that each $\partial R_{i_{1}}$ is identified with one of the 2-spheres in the "boundary" of $Z_{1}$. We also require that the diameters of the sets $R_{i_{1}}$ converge to 0 , so that $\left\{R_{i_{1}}\right\}_{i_{1} \in N}$ is a null family. The action $\angle p^{\prime}$ of $I^{*}$ on $R$ gives rise to an action $\varphi_{l_{1}}$ on each $R_{i_{1}}$ with Fix $\left(\varphi_{l_{1}}\right)=\partial R_{i_{1}}$. We get a space $P_{1}=Z_{1} \cup \bigcup_{i_{1} \in N} R_{i_{1}}$ and an action $\varphi_{1}^{\prime}$ of $I^{*}$ on $P_{1}$ with $\varphi_{1}^{\prime} \mid R_{i_{1}}=\varphi_{i_{1}}$ and Fix $\left(\varphi_{1}^{\prime}\right)=Z_{1}$. Then from each manifold $R_{i_{1}} \backslash \partial R_{i_{1}}$ we remove the union of interiors of a dense, countable null family of 3-cells. This time we also require that this family be $\varphi_{i_{1}}$-invariant. We get a new space $Z_{2}$ and a countable number of 2-spheres in the boundary, and to each of the 2-spheres contained in $R_{i_{1}}, i_{1} \in N$, we attach a copy $R_{i_{1}, i_{2}}$ of $R$. Of course we must ensure that $\left\{R_{i_{1}, i_{2}}\right\}$ is a null family for every $i_{1} \in N$. Again the action $\varphi^{\prime}$ of $I^{*}$ on $R$ gives rise to an action $\varphi_{i_{1}, i_{2}}$ of $I^{*}$ on every $R_{i_{1}, i_{2}}$ with $\operatorname{Fix}\left(\varphi_{i_{1}, i_{2}}\right)=\partial R_{i_{1}, i_{2}}$. These actions, together with the actions $\varphi_{i_{1}}, i_{1} \in N$, give an effective action $\varphi_{2}^{\prime}$ of $I^{*} \times I^{*}$ on the space $P_{2}=Z_{2} \cup \bigcup_{i_{1}, i_{2} \in N} R_{i_{1}, i_{2}}$.

We continue the same procedure infinitely many times, obtaining the spaces $P_{n}$ with an effective action $\varphi_{n}^{\prime}$ of $I^{*} \times \ldots \times I^{*}$. Then we put $P=\lim _{\leftarrow}\left\{P_{n}, \alpha_{n, n+1}\right\}$, where $\alpha_{n, n+1}$ is equal to id on $Z_{n+1}$. It is easy to see that we have a natural action $\varphi_{\infty}$ of $A_{\infty}$ on $P$, where

$$
A_{t x}=\lim \left\{\left(I^{*}\right)^{n}, P_{n, n+1}\right\}, \quad\left(I^{*}\right)^{n}=I^{*} \times \ldots \times I^{*}
$$

and $p_{n, n+1}:\left(I^{*}\right)^{n+1} \rightarrow\left(I^{*}\right)^{n}$ is given by $p_{n, n+1}\left(q_{1}, \ldots, q_{n}, q_{n+1}\right)=\left(q_{1}, \ldots, q_{n}\right)$.

It follows from the commutativity of (10.1) that the orbit space of $\varphi_{\infty}$ is homeomorphic to $X\left(S^{3},\left\{H^{3}\right\}\right)$, where $H^{3}$ is the homology sphere of Poincaré.

11. An uncountable family of homogeneous cohomology 3-manifolds. Let now $\mathscr{M}^{3}$ be a countable family of 3-dimensional irreducible homology spheres such that no two elements of $\mathscr{M}^{3}$ have the same fundamental group. There is an abundance of such 
families. Brieskorn homology spheres [M2] can serve as a good example. The aim of this section is to prove the following theorem, yielding the existence of uncountably many different spaces $X\left(S^{3}, \mathscr{M}^{3}\right)$ which are cohomology manifolds.

Theorem (11.1). If $\mathscr{M}^{\prime}$ and $\mathscr{M}^{\prime \prime}$ are two subfamilies of $\mathscr{M}^{3}$ and $\mathscr{M}^{\prime} \neq \mathscr{M}^{\prime \prime}$ then the spaces $X^{\prime}=X\left(S^{3}, \mathscr{M}^{\prime}\right)$ and $X^{\prime \prime}=X\left(S^{3}, \mathscr{M}^{\prime \prime}\right)$ are not homeomorphic:

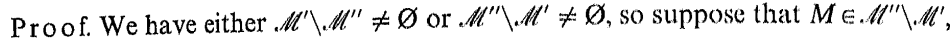
and let $\left\{\mathscr{M}^{\prime}, L_{i}^{\prime}, \alpha_{i, i+1}^{\prime}, \Omega_{i}^{\prime}\right\}$ and $\left\{\mathscr{M}^{\prime \prime}, L_{i}^{\prime \prime}, \alpha_{i, i+1}^{\prime \prime}, \Omega_{i}^{\prime \prime}\right\}$ be defining systems for $X^{\prime}$ and $X^{\prime \prime}$ respectively.

Suppose that $X^{\prime}$ and $X^{\prime \prime}$ are homeomorphic, and consequently have the same Borsuk shape ([B] or [M-S]). That means that there exist increasing maps $\gamma^{\prime}: N \rightarrow N$ $\gamma^{\prime \prime}: N \rightarrow N$ of the set $N$ of positive integers, and families of maps

$$
\underline{f}^{\prime}=\left\{f_{n}^{\prime}: L_{\gamma^{\prime}(n)}^{\prime} \rightarrow L_{n}^{\prime \prime}\right\}_{n \in N} \text { and } \quad \underline{f}^{\prime \prime}=\left\{f_{n}^{\prime}: L_{\gamma^{\prime \prime}(n)}^{\prime \prime} \rightarrow L_{n}^{\prime}\right\}_{n \in N}^{\prime}
$$

such that $f_{k}^{\prime} \alpha_{y^{\prime}(k) \cdot \gamma^{\prime}(k+1)}^{\prime} \simeq \alpha_{k, k+1}^{\prime \prime} f_{k+1}^{\prime}, f_{k}^{\prime \prime} \alpha_{\gamma^{\prime \prime}(k) \cdot i^{\prime \prime}(k+1)}^{\prime \prime} \simeq \alpha_{k, k+1}^{\prime} f_{k+1}^{\prime \prime}$ for any $k \in N$, and for every $i \in N$ there exists $k \in N$ such that $f_{i}^{\prime \prime} f_{\gamma^{\prime}(i)}^{\prime \prime} \alpha_{\gamma^{\prime \prime}\left(\gamma^{\prime}(i)\right), k}^{\prime \prime} \simeq \alpha_{i, k}^{\prime \prime}$ and $f_{i}^{\prime \prime} f_{\gamma^{\prime \prime}(i)}^{\prime \prime} \alpha_{\gamma^{\prime}\left(\gamma^{\prime \prime}(i)\right), k}^{\prime} \simeq \alpha_{i, k}^{\prime}$.

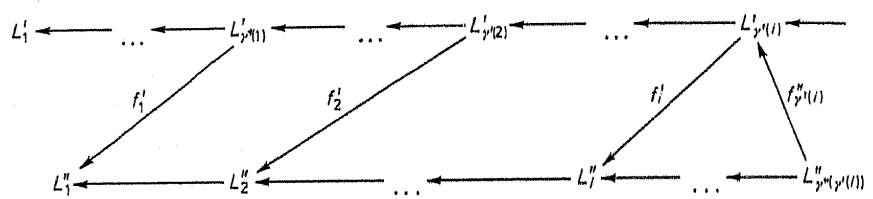

Take any $i$ and $k>i$ such that the last two homotopies hold. Then $L_{k}^{\prime \prime}=L_{i}^{\prime \prime} \# P$ for some homology manifold $P$, and consequently $\pi_{1}\left(L_{k}^{\prime \prime}\right)=\pi_{1}\left(L_{i}^{\prime \prime}\right) * \pi_{1}(P)$ (the choice of base points is irrelevent in our discussion). The map

$$
\alpha=\left(\alpha_{i, k}\right)_{\#:}: \pi_{1}\left(L_{k}^{\prime \prime}\right) \rightarrow \pi_{1}\left(L_{i}^{\prime \prime}\right)
$$

is a contraction of $\pi_{1}\left(L_{i}^{\prime \prime}\right) * \pi_{1}(P)$ onto $\pi_{1}\left(L_{i}^{\prime \prime}\right)$ given by $\alpha\left(a_{1} b_{1} a_{2} b_{2} \ldots\right)=a_{1} a_{2} \ldots$, and of

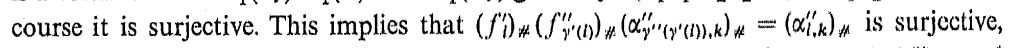
and consequently $\left(f_{i}^{\prime}\right)_{*}$ is surjective. So we have proved that for any $i,\left(f^{\prime}\right)_{*}$ and analogously $\left(f_{i}^{\prime \prime}\right)_{\#}$ are surjective. Set $H_{M}=\pi_{1}(M)$. Every $L_{i}^{\prime \prime}$ is a connected sum of elements of $\mathscr{M}^{\prime \prime}$, and there must exist an index $i$ such that $L_{i}^{\prime \prime}$ contains a summand homeomorphic to $M$. Again, let $k \in N$ be such that $f_{i}^{\prime \prime} f_{\gamma^{\prime}(l)}^{\prime \prime} \alpha_{\gamma^{\prime \prime}\left(\gamma^{\prime}(l)\right), k}^{\prime \prime} \sim \alpha_{i, k}^{\prime \prime}$; then $L_{i}^{\prime \prime}=M \# \bar{P}$ and let $L_{k}^{\prime \prime}=M \# P \# \bar{P}$, and so $\pi_{1}\left(L_{i}^{\prime \prime}\right)=I_{M}^{*} I_{1}, \pi_{1}\left(I_{k}^{\prime \prime}\right)=I_{M} * H_{*} * \bar{A}$, where $\bar{H}=\pi_{1}(P), \bar{H}=\pi_{1}(\bar{P})$. Set $\pi_{1}\left(L_{\gamma^{\prime}(l)}^{\prime}\right)=G$. Then we have maps

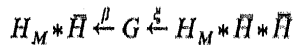

where $\beta=\left(f_{i}^{\prime}\right)_{\#,}, \quad \xi=\left(f_{\gamma^{\prime}(i)}^{\prime \prime}\right)_{; \#}\left(\alpha_{\gamma^{\prime \prime}\left(\gamma^{\prime}(l)\right), k}^{\prime \prime}\right)_{\#}$. As we have mentioned both maps are surjective, and $\alpha=\beta \xi$ is a contraction of $\left(H_{N} * \tilde{H}\right) * \tilde{H}$ onto $H_{M} * H$. Set $G_{M}=\xi\left(H_{M}\right)$ and $\bar{G}=\xi(\bar{H} * \bar{H})$. We have $\alpha \mid H_{M}=\mathrm{id}_{H_{M}}$ and $\beta \mid G_{M}: G_{M} \rightarrow H_{M}$ is an isomorphism. Also $G_{M} \cap \bar{G}=\{1\}$, because $\alpha(\bar{H} * \bar{H})=\beta(G)=\ddot{H}$. The fact that $\xi$ is surjective now implies that $G=G_{M} * \bar{G} \approx H_{M} * \bar{G}$, so $\pi_{1}\left(L_{\gamma^{\prime}(i)}^{\prime}\right) \approx G * H_{M}$. The Kneser conjucture ([H]), yields that $L_{\gamma^{\prime}(i)}^{\prime}=M \# M^{\prime}$ for some closed 3-manifold $M^{\prime}$. But there is no manifold homeomorphic to $M$ in $\mathscr{M}^{\prime}$, so using Milnor's theorem about the uniqueness of a decomposition of a 3-manifold into a connected sum we get a contradiction.

\section{References}

[A-S] F. D. Ancel and L. C. Siebenmann, The construction of homogeneous homology manifolds, Abstracts Amer. Math. Soc. 6 (1985), 92.

[B-P] C. Bessaga and A. Pełczyński, Selected Topics in Infinite-dimensional Topology Monograf. Mat. 58, PWN, Warszawa 1975.

[B] A. Borcl, Seminar on Transformation Groups, Ann. of Math. Stud. 46, Princeton Univ. Press, 1960.

[B] K. Borsuk, Theory of Shape, Monograf. Mat., PWN, Warszawa 1975.

[D] M. W. Davis, Groups generated by reflections and aspherical manifolds not covered by euclidean space, Ann. of Math. (2) 117 (1983), 293-324.

[F] M. H. Freedman, The topology of four dimensional manifolds, J. Differ. Geom. 17 (1982) 357-453.

[H] J. Hempel, 3-Manifolds, Princeton University Press, 1976

[J] W. Jakobsche, The Bing-Borsuk conjecture is stronger than the Poincare conjecture, Fund. Math. 106 (1980), 127-134

[J-R] W. Jakobsche and D. Repovs, An exotic factor of $S^{3} \times R$, Math. Proc. Cambridge Philos. Soc. 107 (1990), 329-344.

[K] R. C. K ir by, Stable homeomorphisms and the annulus conjecture, Ann. of Math. 85 (1969), 575-582.

[K-S] R. C. Kirby and M. G. Scharlemann, Eight faces of the Poincaré homology sphere, in: Geometric Topology, 1979, 113-146.

[Ko] A. Kolmogoroff, Über offene Abbildungen, Ann. of Math. (2) (38) (1937), 36-38.

[M-S] S. Mardešić and J. Segal, Shapes of compacta and ANR-systems, Fund. Math. 72 (1971), 41-59.

[M1] W. S. Massey, Homology and Cohomology Theory, Lecture Notes in Pure and Appl. Math., Dekker, New York and Basel, 1978.

[M2] J. Milnor, On the 3-dimensional Brieskorn manifolds $M(p, q, r)$, in: Knots, Groups and 3-Manifolds, Ann. of Math. Studies, Vol. 84, Princeton Univ. Press., 1975, 175-226. Math., Dekker, New York and Basel, 1978.

[M-Y] D. Montgomery and T. C. Yang, Differentiable transformation groups on homotopy spheres, Michigan Math. J. 14 (1967), 3346.

[P] L. S. Pontryagin, Sur une hypothèse fondamentale de la théorie de la dimension, C. R. Acad. Sci. Paris 190 (1930), 1105-1107.

[Q] F. S. Quin n, Ends of maps III: dimension 4 and 5, J. Differ. Geom. 17 (1982), 503-521.

[S] L. C. Siebenmann, On Gromov's horizon for combinatorially hyperbolic groups, Abstracts Amer. Math. Soc. 6 (1985), 88.

[W] R. F. Willi ams, A useful functor and three famous examples in topology, Trans. Amer. Math. Soc. 106 (1963), 319-329.

INSTITUTE OF MATHEMATICS, UNIVERSITY OF WARSAW PKiN, IX p.

00-901 Warszawa, Poland 